\title{
Parâmetros biofísicos e índices de extremos de temperatura do ar na transição Cerrado-Amazônia
}

\author{
Biophysical parameters and extreme air temperature indexes in the \\ Cerrado-Amazonia transition
}

\author{
Marlus Sabino 1 \\ Jonh Billy da Silva ${ }^{1}$ \\ Rayanna de Oliveira Costa ${ }^{1}$ (iD) \\ Leilane Gomes Duarte ${ }^{1}$ (D) \\ Adilson Pacheco de Souza ${ }^{1,2}$ (D)
}

\begin{abstract}
Palavras-chave:
\section{Resumo}

Albedo

Radiação

Fluxo de calor

Mudanças climáticas

Analisou-se as mudanças de parâmetros biofísicos e tendências de índices de extremos anuais da temperatura do ar na região de Sinop, Mato Grosso, considerando estações meteorológicas automáticas (EMA) instaladas em área urbana e rural. As estimativas das mudanças no uso do solo, albedo da superfície, saldo de radiação $(\mathrm{Rn})$ e fluxos de calor sensível $(\mathrm{H})$ e calor latente (LE) foram obtidas por imagens dos satélites Landsat 8 e Landsat 5, no mês de agosto dos anos de 2007, 2011 e 2017. Com o software Rclimdex, foram calculadas as tendências de seis índices de extremos dos valores máximos, mínimos e médios das temperaturas máximas e mínimas anuais, com calibração pelo método dos mínimos quadrados e significâncias estatísticas consideradas pelo teste de Fisher com nível de significancia igual a a: 0,05. Foram observadas mudanças no uso e ocupação solo e dos parâmetros biofísicos com aumento do albedo e redução do Rn para EMA na área rural, enquanto na estação urbana (UFMT Sinop) observou-se a inversão das parcelas de LE e H. Os índices de extremos indicaram tendência no aumento das temperaturas extremas na área urbana, sendo significativas as mudanças nos índices TNx, DTR, Tn90p e Tx90p. Na última década, com a expansão do município de Sinop-MT, ocorreram alterações nas tendências dos índices de extremos de temperatura, sendo estas, serem atribuídas as mudanças no uso do solo e parâmetros biofísico.
\end{abstract}

\section{Keywords:}

Albedo

Radiation

Heat flux

Climate change

Soil use

\begin{abstract}
This paper aimed to evaluet the changes in the biophysical parameters and trends of the annual extremes of air temperature in Sinop, Mato Grosso, Brazil, using data from automatic meteorological stations (EMA) installed in the rural and urban areas of the municipality. The estimates of changes in soil use, surface albedo, radiation balance $(\mathrm{Rn})$ and sensible heat $(\mathrm{H})$ and latent heat (LE) were obtained by images from the Landsat 8 and Landsat 5
\end{abstract}

${ }^{1}$ Graduate Program in Environmental Physics, Physic Institute, Federal University of Mato Grosso, Cuiabá, Mato Grosso, Brasil.marlussabino@gmail.com; jonhbilly9@gmail.com; rayanna.oli.costa@gmail.com; leilane@fisica.ufmt.br ${ }^{2}$ Institute of Agricultural and Environmental Sciences, Federal University of Mato Grosso (UFMT), Sinop, Mato Grosso, Brasil. pachecoufmt@gmail.com 
satellites in August of the years of 2007, 2011 and 2017. With the Rclimdex software, the trends of six extreme indices of the maximum, minimum and mean values of the annual maximum and minimum temperatures were calculated with least squares calibration and statistical significance considering the Fisher test with significance level equal to a: 0.05 . It was observed changes in soil use and biophysical parameters like albedo increase and reduction of $\mathrm{Rn}$ in the rural area, while in the urban station (UFMT Sinop) occurred inversion of the ration of LE and H. The extreme indexes indicated a tendency of increase in the extreme temperatures in the urban area, being significant the changes in the indices TNx, DTR, Tn90p and Tx90p. In the last decade, with the expansion of the municipality of Sinop-MT, there have been changes in trends of temperature extremes indices, which are attributed to changes in soil use and biophysical parameters.

\section{INTRODUÇÃO}

Nos últimos anos, as evidências do aquecimento global antropogênico passaram a ganhar força com o desenvolvimento de modelos numéricos para predição do aumento na temperatura do ar devido as emissões de gases do efeito estufa. Contudo, a previsão do clima a longo prazo ainda é incerta, existindo divergências sobre os efeitos precisos das mudanças do ambiente no clima (JUNGES; MASSONI, 2018).

As florestas tropicais são ecossistemas que desempenham papel chave na regulação do clima em escala local e regional, em função das interações físicas e biológicas no sistema soloplanta-atmosfera, como a regulação do ciclo do carbono e a disponibilização de água para a atmosfera pelos processos de evapotranspiração (ARTAXO et al., 2014; DOUGHTY et al., 2015). Entretanto, esse equilíbrio dinâmico, principalmente em regiões de transição, está sujeito a força de ações antrópicas como o crescimento dos centros urbanos e manejo da terra, associados às queimadas e ao desmatamento (NOBRE et al., 2007; ARAÚJO et al., 2015).

A substituição de florestas naturais por monoculturas e centros urbanos pode provocar alterações no clima local e regional devido às mudanças no albedo da superfície e das trocas líquidas de radiação e energia entre a superfície e a atmosfera (MARTINS et al., 2015), causando alterações na temperatura do ar e do solo, e na evapotranspiração.

A temperatura do ar é uma variável ambiental que possui maior facilidade de obtenção quando comparada com outras variáveis meteorológicas, e consequentemente, vêm sendo utilizada com maior frequência na identificação de mudanças climáticas pela associação de suas flutuações com a variabilidade de tendências de índices de eventos extremos atribuídas ao aquecimento global (MARENGO et al., 2007). No Brasil, diversos estudos têm utilizado estimativas dos índices de extremos de temperatura, elaborados pela Organização Meteorológica Mundial (OMM), para avaliação das mudanças climáticas locais (DANTAS et al., 2015; SANTOS et al., 2012; SANTOS et al., 2020; SILVA et al., 2015; SILVA et al., 2017). Porém, a maior parte destes estudos concentram-se na região Sul e Sudeste do país, já que essas regiões apresentam maior quantidade de estações meteorológicas e séries com melhor qualidade e intervalo de dados.

A cidade de Sinop é resultado da política de ocupação da Amazônia Legal Brasileira, desenvolvida pelo Governo Federal na década de 1970 e apresenta 139.938 habitantes (IBGE, 2020). Está localizada na região Norte de Matogrosso, com uma áea de $3.194,4 \mathrm{~km}^{2}$ e um perímetro urbano de $17,0 \mathrm{~km}^{2}$. Inseri-se na bacia hidrográfica do Rio Teles Pires (divisor entre a região do Alto e Médio Teles Pires) e apresenta como destaques econômicos os setores agropecuário, extrativista vegetal (madeira), agroindustrial, educação e serviços, sendo ainda, considerada como polo comercial regional de atacado e varejo.

Sinop é tida como uma cidade planejada, observando critérios urbanísticos modernos, com traçado regular e uma área verde média de 27,00 $\mathrm{m}^{2}$ por habitante. $\mathrm{O}$ vertiginoso e recente crescimento do município vem propiciando alterações na ocupação do solo, e consequentemente em parâmetros biofísicos regionais.

Nesse contexto, este estudo objetivou avaliar as mudanças nos parâmetros biofísicos (albedo, 
saldo de radiação, fluxo de calor latente e fluxo de calor sensível), assim como do uso e ocupação solo, e suas implicações nas tendências de índices de extremos climáticos de temperatura do ar, na área urbana e rural do município de Sinop, localizado na região de transição CerradoAmazônia, Norte do Mato Grosso.

\section{MATERIAL E MÉTODOS}

\section{Local de estudo}

As séries de dados de temperaturas máxima e mínima diárias foram obtidas da estação meteorológica automática código A917 pertencente ao Instituto Nacional de Meteorologia (INMET, 2018), instalada na latitude $-11^{\circ} 58^{\prime}$, longitude $-55^{\circ} 34^{\prime}$ e altitude 367 m, e da Universidade Federal do Mato Grosso (UFMT - Campus Universitário de Sinop), instalada latitude $-11^{\circ} 51^{\prime}$, longitude $-55^{\circ} 29^{\prime}$ e altitude $371 \mathrm{~m}$. As estações estão localizadas, respectivamente, no perímetro rural e urbano do município de Sinop - MT; com base de dados compreendendo os anos de 2007-2017 (INMET) e 2011-2016 (UFMT) (Figura 1).

De acordo com a classificação climática de
Koppen, o clima da região é do tipo Aw - tropical úmido, com a estação seca bem definida (maio a setembro), média anual de precipitação em torno de $1970 \mathrm{~mm}$ e temperatura média anual de 24,70 ${ }^{\circ} \mathrm{C}$ (SOUZA et al., 2013) (Figura 2).

\section{Aquisição e processamento das imagens}

A avaliação de mudança do uso e ocupação do solo, assim como, alterações no albedo (a), saldo de radiação $(\mathrm{Rn})$, fluxo de calor latente (LE) e fluxo de calor sensível $(\mathrm{H})$ foram realizadas, com auxílio de feramentas de sensoriamento remoto, utilizando-se seis imagens de refletância da superfície e temperatura de brilho do Operational Land Imager (OLI), Thermal Infrared Sensor (TIRS) e Thematic Mapper (TM), geradas a bordo dos satélites Landsat 8 e Landsat 5, na órbita 226 e ponto 68 e na órbita 227 e ponto 68 , obtidas junto à U. S. Geological Survey (USGS, 2018). Visto que a presença de nuvens nos produtos de sensoriamento podem acarretar erros nas estimativas de fluxos de energia, foram utilizadas imagens correspondentes apenas ao mês de agosto (período de estiagem) dos anos de 2007, 2011 e 2017.

Figura 1. Localização das estações meteorológica automática do INMET e UFMT, Sinop - MT, Brasil.

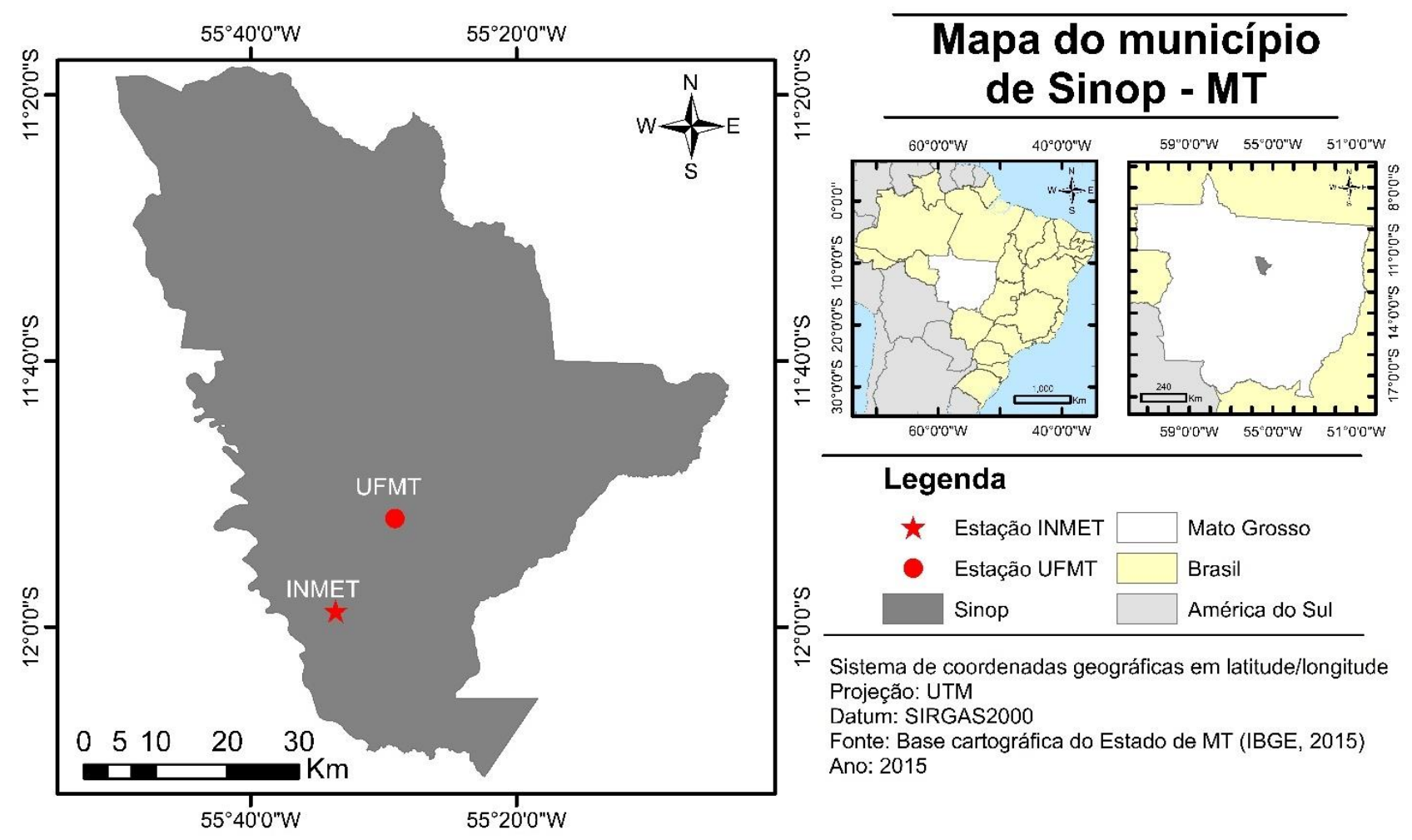

Org.: Dos autores, 2019. 
Figura 2. Climograma da estação Gleba Celeste localizada na região médio norte de Mato Grosso (normal climatológica: 1972-2010).

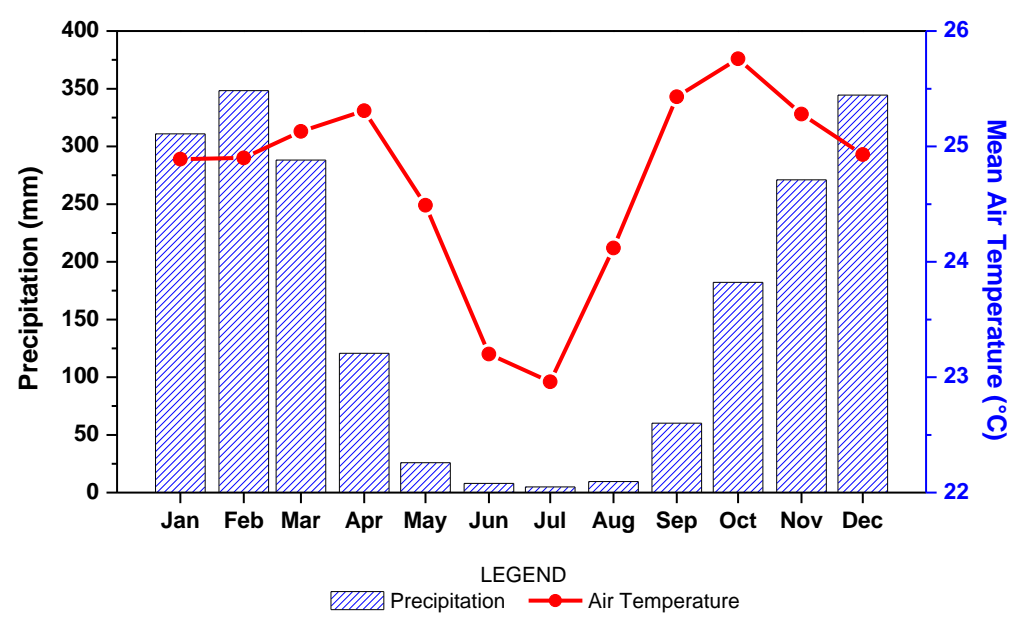

Org.: Adaptado de Souza et al. (2013)

As imagens da refletância da superfície passaram por correção radiométrica, correção atmosférica, correção geométrica sistemática, correção de precisão usando chips de controle de chão e o uso de um modelo digital de elevação para corrigir erro de paralaxe devido ao relevo topográfico local (CLAVERIE et al., 2015).

\section{Estimativas dos parâmetros biofísicos}

As estimativas de albedo, saldo de radiação, fluxo de calor sensível e fluxo de calor latente foram obtidas como parte do Algoritmo R-SSEB (Simplified Modeling for Energy Balance Estimation to the Regional Scale Surface) proposto por Araújo et al. (2017). O albedo da superfície foi calculado de acordo com a Equação 1, proposta por Tasumi et al. (2008).

$\alpha_{\text {sup }}=\frac{\alpha_{t o a}-\alpha_{a t m}}{\tau_{s w}^{2}}$

em que: $\alpha_{a t m}$ é a porção da radiação solar refletida pela atmosfera, adotou-se 0,03 de acordo com Bastiaanssen (2000); $\tau_{s w}^{2}$ é a transmitância atmosférica; e $\alpha_{\text {toa }}$ representa 0 albedo da superfície, sem correção atmosférica.

O albedo da superfície, sem correção atmosférica, foi obtido através de combinação linear das refletâncias espectrais das bandas $(\rho)$ 2, 3, 4, 5, 6 e 7, Equação 2. A transmitância atmosférica para dias de céu claro, Equação 3, foi determinada conforme proposto por Allen et al. (2007).

$$
\begin{aligned}
\alpha_{\text {toa }}=0,300_{\rho 2}+0, & 277_{\rho 3}+0,233_{\rho 4}+0,143_{\rho 5}+0,036_{\rho 6} \\
+ & 0,012_{\rho 7}
\end{aligned}
$$

em que: $P$ é a pressão atmosférica $\mathrm{kPa} ; W$ é água precipitável na atmosfera; $\cos \theta_{\text {hor }}$ é ângulo zenital solar sobre uma superfície horizontal; $\mathrm{K} t$ é o coeficiente de turbidez ( $K \mathrm{t}=1,0$ para ar limpo e $K t=0,5$ para ar extremamente turvo, empoeirado ou poluído).

O NDVI (Normalized Difference Vegetation Index) que serve como medida de avaliação da condição da vegetação é dado pela Equação 4, conforme proposto por Huete et al. (2002).

$N D V I=\frac{\rho_{I V P}-\rho_{V E R}}{\rho_{I V P}+\rho_{V E R}}$

em que: $\rho_{I V P}$ é a refletância do infravermelho próximo; e $\rho_{V E R}$ é a refletância do vermelho

O saldo de radiação diário $\left(R N_{24}\right)$, representa a contabilização dos fluxos radiativos à superfície em 24 horas, pode ser obtido através do cômputo dos fluxos ascendentes e descendentes das radiações de onda longa e curta atuantes no sistema solo-planta-atmosfera, Equação 5.

$R n=R_{S \downarrow}\left(1-\alpha_{\text {sup }}\right)-R_{L \uparrow}+R_{L \downarrow}-\left(1-\varepsilon_{0}\right) R_{L \downarrow}$

em que: $R_{S} \downarrow$ é a radiação de ondas curtas 
incidente $\left(\mathrm{Wm}^{-2}\right), \alpha_{\text {sup }}$ é o albedo da superfície (adimensional), $R_{L} \downarrow$ é a radiação de ondas longas emitidas pela atmosfera na direção da superfície $\left(\mathrm{Wm}^{-2}\right), \quad R_{L \uparrow}$ é a radiação de ondas longas emitidas pela superfície; e $\varepsilon_{0}$ é a emissividade.

Visto que a radiação solar de onda curta incidente $\left(R_{S} \downarrow\right)$ é a principal fonte de energia para a ET, a Equação 5 pode ser simplificada conforme Equação 6, na qual, a radiação global representa a soma da radiação de onda curta, direta e difusa, que alcança a superfície da Terra $\left(\mathrm{Wm}^{-2}\right)$, sendo atenuada, juntamente com os componentes de onda longa, pelas propriedades da transmissividade atmosférica e albedo da superfície.

$R n_{24}=R g_{24} \times\left(1-\alpha_{\text {sup }}\right)-\left(110 \times \tau_{24}\right)$

em que: $R g_{24}$ é a radiação global diária (W m-2) obtida de uma estação meteorológica automática integrante da rede do INMET próxima a área de estudo; $\alpha_{\text {sup }}$ é o albedo da superfície; e $\tau_{24}$ é a transmissividade média diária da atmosfera.

As componentes do balanço de energia são obtidas a partir estimativa do $\mathrm{Rn}$ descrita anteriormente. Inicialmente, calculou-se o fluxo de calor no solo $(\mathrm{G})$ através de parametrizações. Em seguida procedeu-se a determinação dos fluxos de calor sensível $(\mathrm{H})$ e de calor latente (LE), os quais são estimados por meio da fração evaporativa (FE).

O valor de G foi computado segundo a Eq. (7) desenvolvida por Bastiaanssen (2000):

$G=\left[\frac{T_{s}}{\alpha}\left(0,0038 \alpha+0,0074 \alpha^{2}\right)\left(1-0,98 N D V I^{4}\right)\right] R_{n}$

em que: $\mathrm{T}_{\mathrm{S}}$ é temperatura da superfície $\left({ }^{\circ} \mathrm{C}\right) ;$ a é o albedo da superfície (adimensional); NDVI é o índice de vegetação (adimensional); Rn é o saldo de radiação $\left(\mathrm{Wm}^{-2}\right)$.

No modelo R-SSEB a fração evaporativa (FE) é calculada utilizando a média de pelo menos três pixels quentes e três pixels frios identificados por meio de cartas de NDVI e Ts (SENAY et al., 2007). Partindo do pressuposto de que os pixels quentes apresentam valores pequenos de ET e os pixels frios representam a ET máxima ao longo de toda área de estudo, a média de cada conjunto de pixels pode ser utilizada para calcular a FE para todos os pixels da cena a partir da Equação 8 desenvolvida por Senay et al. (2013):
$F E=\frac{T_{H}-T_{S}}{T_{H}-T_{C}}$

em que: $T_{H}$ é a média dos pixels quentes, $T_{C}$ é a média dos pixels frios e $T_{S}$ é a temperatura da superfície.

Uma vez determinada a $\mathrm{FE}$, os fluxos de calor sensível (H) e latente (LE) foram calculados conforme as Eqs. (9) e (10) (ROERINK et al., 2000; SOBRINO et al., 2007):

$H=(1-F E)(R n-G)$

$L E=F E(R n-G)$

As médias e intervalos de confiança de $95 \%$ dos parâmetros biofísicos (a, Rn, H, LE) foram calculadas por bootstrapping de 1000 iterações das reamostragens aleatórias com substituição (EFRON; TIBSHIRANI, 1993) e comparadas pelo teste de teste de Kruskal-Wallis, $\alpha=0,05$.

\section{Índices de extremos de temperatura do ar}

Foram calculados 11 índices de extremos de temperatura do ar (Tabela 1), com o software RClimdex, desenvolvido para o $\mathrm{R}$ pelo National Climate Data Centre (NCDC) da National Oceanic and Atmospheric Administration (NOAA) conforme documentado por Easterling et al. (2003); Tank et al. (2009), por ser recomendado na análises de índices extremos climáticos que auxiliam no monitoramento e na deteç̧ão de mudanças climáticas.

Um pré-requisito para o cálculo dos índices é o controle de qualidade dos dados (ZHANG; YANG, 2004), que procede-se em 3 etapas: a) substituição de dados faltosos; b) substituição de valores não representativos (Tmax < Tmin); e c) identificação de valores extremos (outliers) com base em número pré-determinado de desvios padrões.

Após o teste de controle de qualidade os dados passam ainda por análise de homogeneidade de forma a detectar possíveis descontinuidades ou deslocamentos nos registros de dados das séries temporais. $O$ teste de homogeneidade é baseado no teste t com máxima penalizada (WANG et al., 2007) e no teste F com máxima penalizada (WANG, 2008), que estão embutidos em um algoritmo de teste recursivo. As tendências anuais dos índices de extremos climáticos foram obtidas por análises de regressão pelo método dos mínimos quadrados e 
a significância estatística da tendência pelo teste

de Fisher.

Tabela 1. Índices de extremos de temperatura do ar avaliados na regiao de Sinop, MT.

\begin{tabular}{clc}
\hline Índice & Definição & Unidade \\
\hline Tn10p & Porcentagem anual de dias em que TN < percentil 10 & $\%$ \\
Tx10p & Porcentagem anual de dias em que TX < percentil 10 & $\%$ \\
Tn90p & Porcentagem anual de dias em que TN $>$ percentil 90 & $\%$ \\
Tx90p & Porcentagem anual de dias em que TX > percentil 90 & $\%$ \\
CSDI & Número máximo de dias consecutivos no ano com TN $<$ percentil 10 & Dias \\
WSDI & Número máximo de dias consecutivos no ano com TX > percentil 90 & Dias \\
TXx & Valor máximo anual da temperatura máxima diária & ${ }^{\circ} \mathrm{C}$ \\
TXn & Valor mínimo anual da temperatura máxima & ${ }^{\circ} \mathrm{C}$ \\
TNx & Valor máximo anual da temperatura mínima diária & ${ }^{\circ} \mathrm{C}$ \\
TNn & Valor mínimo anual da temperatura mínima diária & ${ }^{\circ} \mathrm{C}$ \\
DTR & Diferença média anual entre TX e TN & ${ }^{\circ} \mathrm{C}$ \\
\hline
\end{tabular}

Org.: Dos autores, 2019.

\section{RESULTADOS E DISCUSSÃO}

A análise da mudança no uso e ocupação do solo demonstraram gradual redução nas áreas ocupadas por florestas nativas, que passaram de $41,76 \%$ para $39,47 \%$ da área total do município, assim como, aumento das áreas agricultáveis, cujo percentual passou de $55,51 \%$ para $56,22 \%$. Contudo, a principal mudança ocorreu devido ao crescimento do centro urbano, com aumento de $408 \%$ em uma década, sendo o crescimento mais acentuado entre 2011 e 2017 (Tabela 2; Figura $3)$.

Tabela 2. Porcentagem de área de diferentes uso e ocupação do solo, entre os anos de 2007 e 2017, do município de Sinop - MT.

\begin{tabular}{lccc}
\hline \multirow{2}{*}{ Uso e Ocupação } & \multicolumn{3}{c}{ Ano } \\
\cline { 2 - 4 } & 2007 & 2011 & 2017 \\
\hline Área urbana & 0,71 & 1,05 & 3,61 \\
Solo exposto / Agricultura & 55,51 & 57,74 & 56,22 \\
Floresta & 41,76 & 39,96 & 39,47 \\
\hline
\end{tabular}

Org.: Dos autores, 2019.

Os parâmetros biofísicos, semelhantemente ao uso e ocupação do solo, também sofreram modificações, principalmente na área urbana. O albedo próximo à estação da UFMT aumentou de 0,25 para 0,45 entre 2007 e 2017, sendo este valor em torno de $31 \%$ superior ao encontrado na estação INMET em 2017 (área rural). Inversamente ao albedo, o saldo de radiação, no período avaliado, apresentou redução de 30,78\% e $14,29 \%$ para a estação UFMT e INMET, respectivamente (Tabela 3 ).

$\mathrm{Na}$ estação do INMET as parcelas dos fluxos de energia mantiveram-se semelhantes entre o período de estudo, correspondendo, para o fluxo de calor latente, a 52,60\% (2007) e 53,91\% (2017) do saldo de radiação, e, para o fluxo de calor sensível, a 35,04\% (2007) e 34,40\% (2017). O processo de urbanização, contudo, foi responsável por alterações no particionamento de energia destinados aos fluxos, notando-se, para a estação da UFMT, aumento da partição de fluxo de calor sensível, que passou de 28,75\% (2007) para $53,94 \%$ (2017), e redução do fluxo de calor latente, cuja porcentagem decresceu de 58,99\% (2007) para 25,23\% (2017) (Tabela 3).

Os resultados dos índices de extremos de temperatura do ar nas estações meteorológicas são apresentadas na Figura 4. Na estação do INMET não foram encontradas tendências significativas ( $\mathrm{p}$-valor $<0,10)$ nos índices relacionados a mudanças no valor das temperaturas máximas e mínimas (TXx, TXn, TNx, TNn e DTR). Contudo, na estação da UFMT, foram significativas as tendências de aumento de $0,34^{\circ} \mathrm{C}$ nos máximos da temperatura mínima do ar $(\mathrm{TNx})$ e de $0,32{ }^{\circ} \mathrm{C}$ na amplitude diária (DTR). 
Figura 3. Mapa de uso e ocupação do solo do município de Sinop - MT dos anos de 2007, 2011 e 2017.
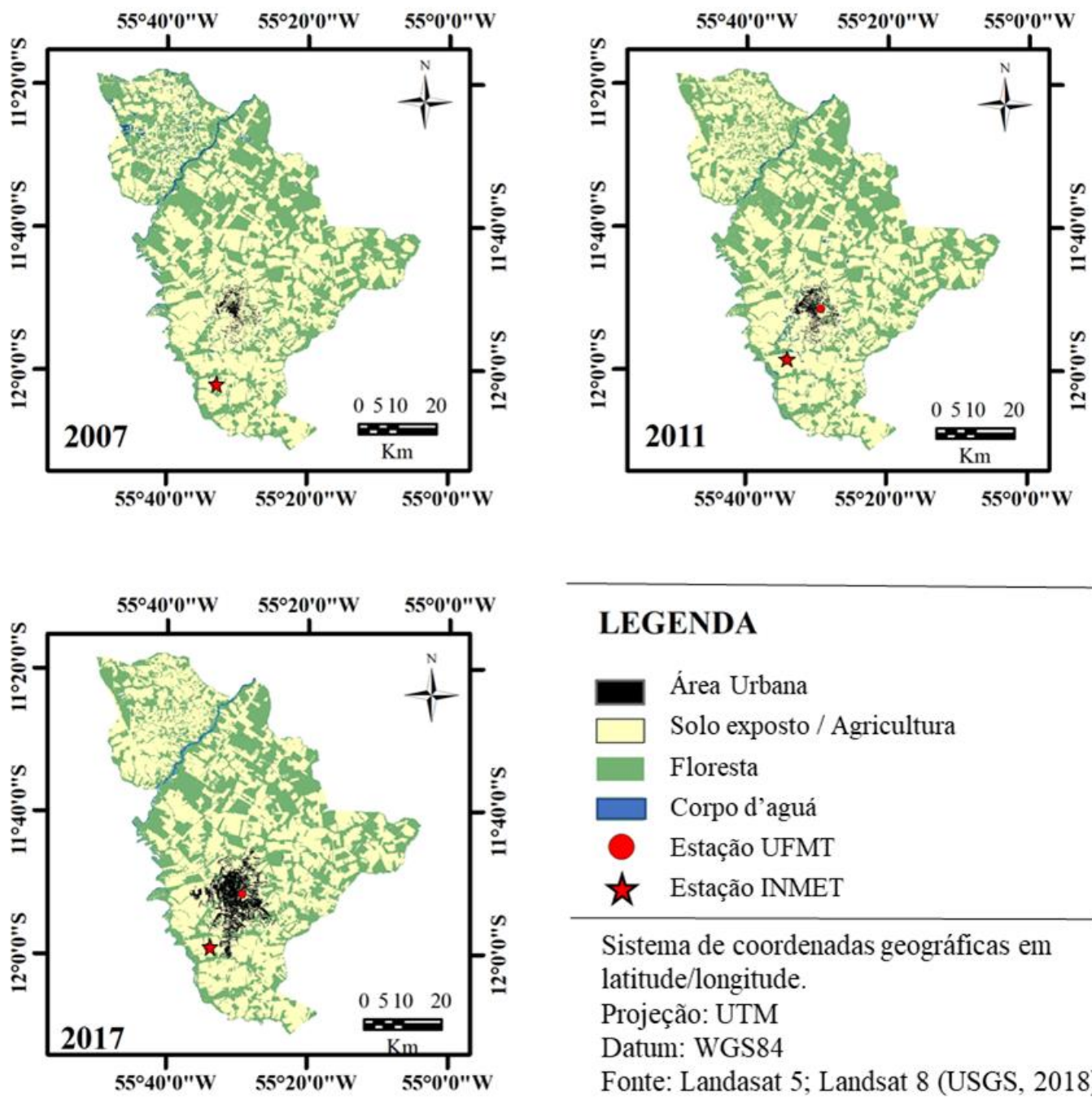

\section{LEGENDA}

- Área Urbana

Solo exposto / Agricultura

Floresta

Corpo d'aguá

- Estação UFMT

Ł Estação INMET

Sistema de coordenadas geográficas em latitude/longitude.

Projeção: UTM

Datum: WGS84

Fonte: Landasat 5; Landsat 8 (USGS, 2018)

Org.: Dos autores, 2019.

Tabela 3. Porcentagem de área de diferentes uso e ocupação do solo, entre os anos de 2007 e 2017, do

\begin{tabular}{ccccccc}
\multicolumn{7}{c}{ município de Sinop-MT. } \\
\hline \multirow{2}{*}{ Variável } & 2007 & 2011 & 2017 & 2007 & 2011 & 2017 \\
\cline { 2 - 7 } & 0,25 & 0,43 & 0,45 & 0,20 & 0,24 & 0,31 \\
\multirow{2}{*}{$\mathrm{a}$} & $(0,1)$ & $(0,1)$ & $(0,1)$ & $(0,1)$ & $(0,1)$ & $(0,1)$ \\
& 1862,3 & 1330,8 & 1289,0 & 1875,5 & 1621,2 & 1607,4 \\
$\mathrm{Rn}$ & $(17,2)$ & $(17,2)$ & $(17,2)$ & $(16,9)$ & $(16,9)$ & $(16,9)$ \\
& 1098,6 & 419,1 & 325,2 & 986,5 & 767,2 & 866,5 \\
$\mathrm{LE}$ & $(23,2)$ & $(23,2)$ & $(23,2)$ & $(22,8)$ & $(22,8)$ & $(22,8)$ \\
& 535,5 & 648,2 & 695,3 & 657,2 & 618,7 & 553,0 \\
$\mathrm{H}$ & $(12,1)$ & $(12,1)$ & $(12,1)$ & $(11,9)$ & $(11,9)$ & $(11,9)$ \\
\hline
\end{tabular}

Valores entre parênteses representam a diferença entre o intervalo de confiança e a média. Org.: Dos autores, 2019. 
Figura 4. Índices extremos de temperaturas máxima e mínima do ar nas estações meteorológicas do INMET e UFMT.

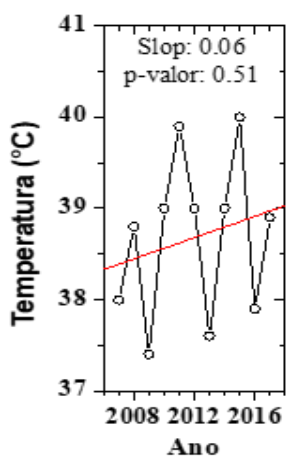

INMET - TXX

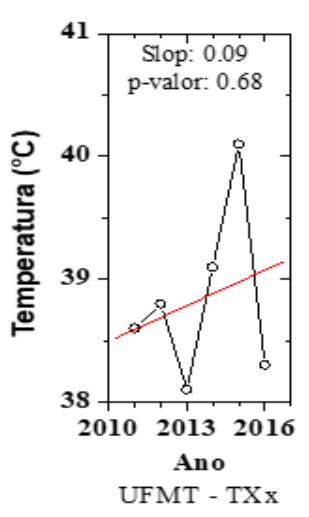

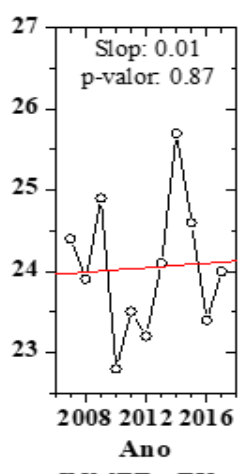

INMET - TXn

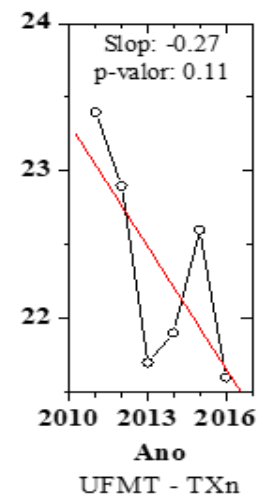

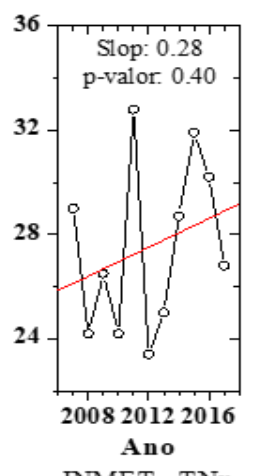

INMET - TNX

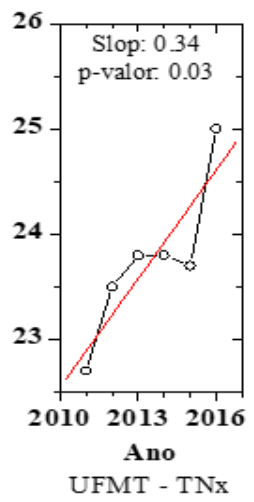

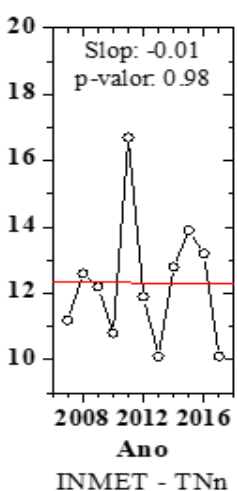

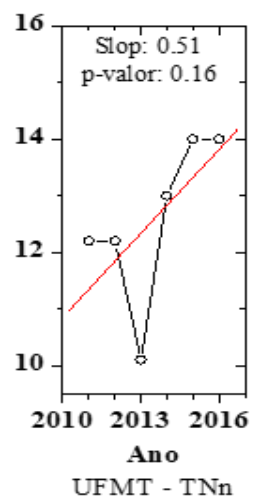

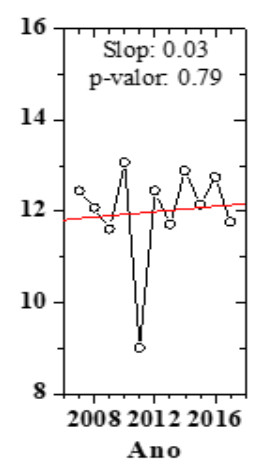

INMET - DTR

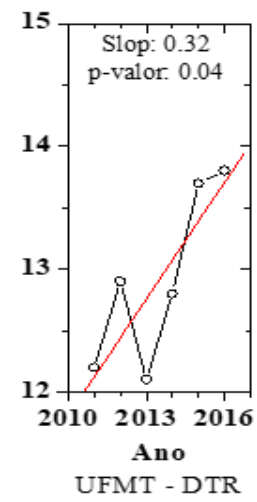

Org.: Dos autores, 2019.

Quanto as alterações nos dias de ocorrência de temperaturas extremas observou-se, na estação do INMET, tendências de aumento de dias com temperaturas máximas e mínimas superiores, dadas pelo índices Tx90p (0,91\%) e Tn90p (1,15\%). Tais alterações são mais evidentes na estação UFMT, na qual, observa-se aumentos de 4,84\% (Tn90p) e 3,15\% (Tx90p) (Figura 5).

Tendências de aumento de índices relacionados à temperatura também foram encontrados por Marengo e Camargo (2008) em 27 estações no sul do Brasil, entre 1960 e 2002, com aumentos de 0,5 a $0,8{ }^{\circ} \mathrm{C}$ decênio ${ }^{-1}$ nas temperaturas mínimas e de $0,4^{\circ} \mathrm{C}$ decênio ${ }^{-1}$ nas máximas, assim como, pelo relatório sintético do IPCC (2007), que apresenta uma visão geral sobre o comportamento das temperaturas médias anuais no continente Sul-Americano e, semelhante ao estudo, mostram tendências de aquecimento de $0,5{ }^{\circ} \mathrm{C}$ no período de 1950 a 2000.

A tendência de aumento da temperatura do ar observado, principalmente, na estação da UFMT está relacionada, entre outros, as mudanças de ocupação do solo. Entre as mudanças no uso do solo, o desmatamento, segundo Foley et al. (2005), é um dos principais contribuintes para a mudança climática. A conversão da vegetação nativa por áreas de atividades agrícolas e centros urbanos são responsáveis por mudanças na temperatura do ambiente, visto que, causam redução dos processos de evapotranspiração (HUNKE et al., 2015), alterando o particionamento e a magnitude do balanço de radiação, albedo e fluxos de calor latente e sensível (RODRIGUES et al., 2013).

A ausência de cobertura vegetal implica, ainda, na maior reflexão da radiação de ondas curtas e maior emissão de radiação de ondas longas, reduzindo o saldo de radiação da superfície (ANDRADE et al., 2014), e faz com que a energia disponível ao ambiente seja utilizada prioritariamente, no fluxo de calor sensível, aquecendo o solo e o ar (BIUDES et al., 2015). Em contrapartida, ambientes vegetados, por utilizarem a maior parte do saldo de radiação nos processos de evapotranspiração (calor latente), tendem a aumentare o conteúdo de vapor d'água disponível no ambiente e reduzir os valores de temperatura máxima. 
Figura 5. Índices relativos ao número de dias com valores extremos de temperaturas máxima e mínima do ar nas estações meteorológicas do INMET e UFMT.
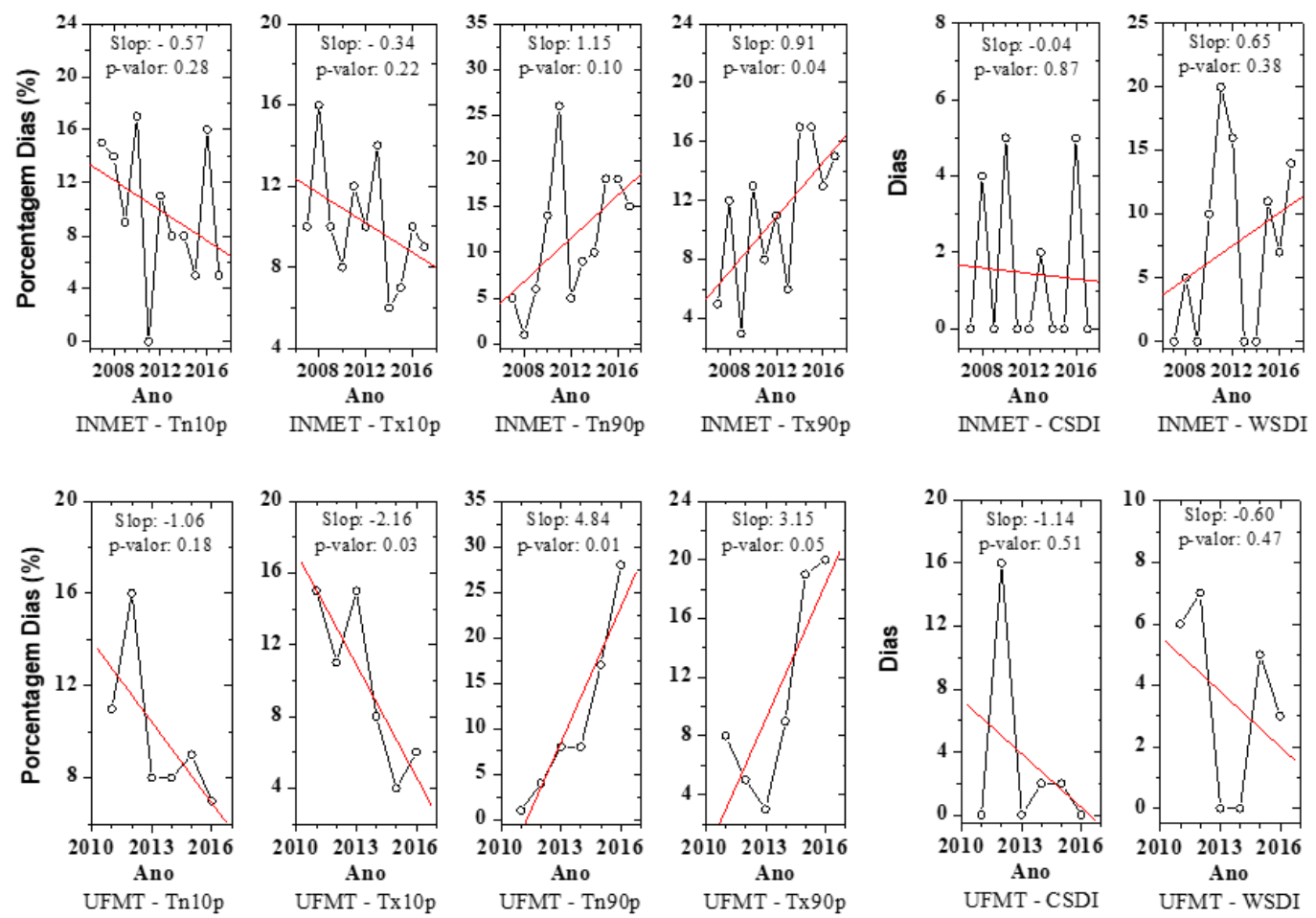

Org.: Dos autores, 2019.

Esses resultados, contudo, fazem parte de uma análise parcial visto que não é possível atribuir as diferenças entre as tendências de temperatura exclusivamente ao uso e ocupação do solo e a expansão das áreas urbanas, além de, a base de dados ainda não corresponder aos 30 anos de medições, recomendado pela OMM, para caracterização climática (SILVA et al., 2017).

\section{CONSIDERAÇÕES FINAIS}

As mudanças no uso e ocupação do solo entre os anos de 2007 e 2017 foram responsáveis pelo aumento de $80 \%$ e $55 \%$ no do albedo superficial e redução de $30,78 \%$ e $14,29 \%$ no saldo de radiação, para o perímetro urbano (UFMT) e rural (INMET) do município de Sinop-MT, respectivamente.

As parcelas de energia destinadas ao $\mathrm{H}$ e LE se mantiveram constantes na estação do perímetro rural (INMET). No perímetro urbano (UFMT) houve inversão das parcelas de energia do saldo de radiação com aumento do $\mathrm{H}$ (aquecimento do ar) e redução do LE.

A estação no perímetro urbano (UFMT) apresentou maiores valores de aumento nos de índices de extremos de temperatura $\mathrm{TNx}(0,34$ $\left.{ }^{\circ} \mathrm{C} / \mathrm{ano}\right)$, DTR (0,32 $\left.{ }^{\circ} \mathrm{C} / \mathrm{ano}\right), \operatorname{Tn} 90 \mathrm{p}$ (4,84\%) e Tx90p (3,15\%), indicando que, a crescente urbanização do município começa a alterar os parâmetros ambientais locais.

$\mathrm{Em}$ análises futuras, recomenda-se a incorporação de outras estações meteorológicas instaladas em outros pontos do município, visto que, a expansão urbana está ocorrendo em diferentes eixos (sentidos).

\section{AGRADECIMENTOS}

Ao Instituto Nacional de Meteorologia (INMET) pela disponibilização das bases de dados. O presente trabalho foi realizado com apoio da Coordenação de Aperfeiçoamento de Pessoal de Nível Superior - Brasil (CAPES) - Código de Financiamento 001

\section{REFERÊNCIAS}

ALLEN, R. G.; TASUMI, M.; TREZZA, R. Satellite-based energy balance for mapping 
evapotranspiration with internalized calibration (METRIC) - Model. Journal of Irrigation and Drainage Engineering, v. 133, p. 380-394, 2007. DOI: 10.1061/(ASCE)0733-9437(2007)133:4(380)

ANDRADE, A. M. D.; MOURA, M. A. L.; SANTOS, A. B.; CARNEIRO, R. G.; JUNIOR, R. S. Radiação fotossintéticamente ativa incidente e refletida acima e abaixo do dossel de Floresta de Mata Atlântica em Coruripe, Alagoas. Revista Brasileira de Meteorologia, v. 29, n. 1, p. 68-79, 2014. https://doi.org/10.1590/S010277862014000100007

ARAÚJO, F. C. D.; SANTOS, C. A. C.; NASCIMENTO, F. C. A. Estudo dos índices extremos de temperatura na bacia hidrográfica do baixo rio Colorado-EUA. Revista Brasileira de Meteorologia, v. 30, n. 1, 2015. https://doi.org/10.1590/0102778620120530

ARAÚJO, A. L.; SILVA M. T.; SILVA, B. B.; SANTOS, C. A. C.; AMORIM, M. R. B. Modelagem Simplificada Para Estimativa do Balanço de Energia à Superfície em Escala Regional (R-SSEB). Revista Brasileira de Meteorologia, v. 32, n. 3, p. 433-446, 2017. https://doi.org/10.1590/0102-77863230010

ARTAXO, P.; DIAS, M. A. F. D. S.; NAGY, L.; LUIZÃO, F. J.; CUNHA, H. B. D.; QUESADA, C. A.; MARENGO, J. A.; KRUSCHE, A. Perspectivas de pesquisas na relação entre clima e o funcionamento da Floresta Amazônica. Ciência e Cultura, v. 66, n. 3, p. 41-46, 2014. http://dx.doi.org/10.21800/S000967252014000300014

BASTIAANSSEN, W. G. M. SEBAL-based sensible and latent heat fluxes in the irrigated Gediz Basin, Turkey. Journal of Hydrology, v. 229, p. 87-100, 2000. https://doi.org/10.1016/S0022-1694(99)002024

BIUDES, M. S.; VOURLITIS, G. L.; MACHADO, N. G.; DE ARRUDA, P. H. Z.; NEVES, G. A. R.; DE ALMEIDA LOBO, F.; NEALE, C. M. U.; NOGUEIRA, J. S. Patterns of energy exchange for tropical ecosystems across a climate gradient in Mato Grosso, Brazil. Agricutural and Forest Meteorology, v. $202, \quad$ p. 112-124, 2015 https://doi.org/10.1016/j.agrformet.2014.12.00 8

CLAVERIE, M.; VERMOTE, E. F.; FRANCH, B.; MASEK, J. G. Evaluation of the Landsat-5 TM and Landsat-7 ETM + surface reflectance products. Remote Sensing of
Environment, v. 169, p. 390-403, 2015. https://doi.org/10.1016/j.rse.2015.08.030

DANTAS, L. G.; SANTOS, C. A. C. D.; OLINDA, R. A. D. Tendências anuais e sazonais nos extremos de temperatura do ar e precipitação em Campina Grande-PB. Revista Brasileira de Meteorologia, v. 30, n. 4, p. 423-434, 2015. https://doi.org/10.1590/0102778620130088

DOUGHTY, C. E.; METCALFE, D. B.; GIRARDIN, C. A.; AMEZQUITA, F. F.; DURAND, L.; HUASCO, W. H.; ESPEJO , J. E. S.; MURAKAMI, A. A.; COSTA, M. C.; COSTA, A. C. L.; ROCHA, W.; MEIR, P.; GALBRAITH, D.; MALHI, Y. Source and sink carbon dynamics and carbon allocation in the Amazon basin. Global Biogeochemical Cycles, v. 29, n. 5, p. 645-655, 2015. https://doi.org/10.1002/2014GB005028

EASTERLING, D. R.; ALEXANDER, L. V.; MOKSSIT, A.; DETEMMERMAN, V. CCI/CLIVAR workshop to develop priority climate indices. Bulletin of the American Meteorological Society, v. 84, n. 10, p. 1403-1407, 2003. DOI: 10.1175/BAMS-84-101403

EFRON, B.; TIBSHIRANI, R. J. An introduction to the bootstrap. New York: Chapman \& Hall; 1993. 443 p.

FOLEY, J. A.; FRIES, R.; ASNER, G. P.; BARFORD, C.; BONAN, G.; CARPENTER, S. R.; CHAPIN, F. S.; COE, M. T.; DAILY, G. C.; GIBBS, H. K.; HELKOWSKI, J. H.; HOLLOWAY, T.; HOWARD, E. A.; KUCHARIK, C. J.; MONFREDA, C.; PATZ, J. A.; PRENTICE, C. RAMANKUTTY, N.; SNYDER, P. K. Global consequences of land use. Science, v. 309, n. 5734, p. 570-574, 2005. DOI: $10.1126 /$ science. 1111772

HUETE, A.; DIDAN, K.; MIURA, T.; RODRIGUEZ, E.P.; GAO, X.; FERREIRA, L. G. Overview of the radiometric and biophysical performance of the MODIS vegetation indices. Remote Sensing of Environment, v. 83, p. 195-213, 2002. https://doi.org/10.1016/S0034-4257(02)000962

HUNKE, P.; ROLLER, R.; ZEILHOFER, P.; SCHRÖDER, B.; MUELLER, E.N. Soil changes under different land-uses in the Cerrado of Mato Grosso, Brazil. Geoderma Regional, v. 4, p. 31-43, 2015. https://doi.org/10.1016/j.geodrs.2014.12.001

IBGE - Instituto Brasileiro de Geografia e Estatística. Cidades e Estados: Sinop (código 5107909). Disponível em: 
$<$ https://www.ibge.gov.br/cidades-eestados/mt/sinop.html>. Acesso em: $30 \mathrm{de}$ julho de 2020.

INMET - Instituto Nacional de Meteorologia. Estação Meteorológica de Observação de Superfície Automática. 2018 Disponível em: <http://www.inmet.gov.br>. Acesso em: 06 de julho de 2018.

INTERGOVERNMENTAL PANEL ON CLIMATE CHANGE - IPCC. Climate Change 2007: Synthesis Report. IPCC Plenary XXVII, Valencia, Spain, 2007. 73 p.

JUNGES, A. L.; MASSONI, N. T. O Consenso Científico sobre Aquecimento Global Antropogênico: Considerações Históricas e Epistemológicas e Reflexões para o Ensino dessa Temática. Revista Brasileira de Pesquisa em Educação em Ciências, v. $18, \quad$ n. $2, \quad$ p. $455-491,2018$. https://doi.org/10.28976/19842686rbpec2018182455.

MARTINS, A. L.; CUNHA, C. R.; RODRIGUES, P. V. M.; MORAIS, D. V. H.; GOMES, M. N.; ALMEIDA, L. F.; BIUDES, M. S. Mudanças em índices biofísicos devido à alteração da cobertura do solo em área nativa de Cerrado em Mato Grosso. Ciência e Natura, v. 37, n. 3 ,

2015.

http://dx.doi.org/10.5902/2179460X16145

MARENGO, J. A.; ALVES, L.; VALVERDE, M.; ROCHA, R.; LABORBE, R. Eventos extremos em cenários regionalizados de clima no Brasil e América do Sul para o Século XXI: Projeções de clima futuro usando três modelos regionais. Relatório 5. São Paulo: Ministério do Meio Ambiente, 2007. 77 p.

MARENGO, J. A.; CAMARGO, C. C. Surface air temperature trends in Southern Brazil for 1960-2002. International Journal of Climatology: Journal of the Royal Meteorological Society, v. 28, p. 893-904, 2008. https://doi.org/10.1002/joc. 1584

NOBRE, C. A.; SAMPAIO, G.; SALAZAR, L. Mudanças climáticas e Amazônia. Ciência e Cultura, v. 59, n. 3, p. 22-27, 2007.

RODRIGUES, T. R.; PAULO, S. R.; NOVAIS, J. W.; CURADO, L. F.; NOGUEIRA, J. S.; DE OLIVEIRA, R. G.; LOBO, F. A.; VOURLITIS, G. L. Temporal patterns of energy balance for a Brazilian tropical savanna under contrasting seasonal conditions.

International Journal of Atmospheric Sciences, v. 2013, p. 1-9, 2013. https://doi.org/10.1155/2013/326010

ROERINK, G. J.; SU, Z.; MENENTI, M. A
Simple Remote Sensing Algorithm to Estimates the Surface Energy Balance. Physics and Chemistry of the Earth, n. $25, \quad$ p. $147-157,2000$. https://doi.org/10.1016/S1464-1909(99)001288

SANTOS, C. A. C. D.; SATYAMURTY, P.; GOMES, O. M.; SILVA, L. E. M. G. D. Variability of extreme climate indices at Rio claro, São Paulo, Brazil. Revista Brasileira de Meteorologia, v. 27, n. 4, p. 395-400, 2012 . https://doi.org/10.1590/S010277862012000400003.

SANTOS, J. R. N., DE ARAÚJO, M. L. S., JUNIOR, C. H. L. S., DOS SANTOS, J. S., ALMEIDA, J. L., LIMA, T. V., SOUZA, L.V. P.; AGUIAR, P. H. M.; SILVA, F. B. Tendências de extremos climáticos na região de transição Amazônia-Cerrado no Estado do Maranhão. Revista Brasileira de Climatologia, v. 26, p. 130-154, 2020. DOI: http://dx.doi.org/10.5380/abclima.v26i0.62883.

SENAY, G. B.; BUDDE, M.; VERDIN, J. P. ; MELESSE, A. M. A coupled remote sensing and simplified surface energy balance approach to estimate actual evapotranspiration from irrigated fields. Sensors, v. 7, p. 979-1000, 2007. https://doi.org/10.3390/s7060979.

SENAY, G. B.; BOHMS, S.; SINGH, R. K.; GOWDA, P. H.; VELPURI, N. M. Operational evapotranspiration mapping using remote sensing and weather datasets: a new parameterization for the SSEB approach. Journal of the American Water Resources Association, v. 49, p. 577- 591, 2013. https://doi.org/10.1111/jawr.12057.

SILVA, R. O. B.; MONTENEGRO, S. M. G. L.; SOUZA, W. M. Tendências de mudanças climáticas na precipitação pluviométrica nas bacias hidrográficas do estado de Pernambuco. Engenharia Sanitária e Ambiental, v. 22, n. 3, p. 579-589, 2017. https://doi.org/10.1590/s141341522017142481.

SILVA, W. L.; DERECZYNSKI, C.; CHANG, M.; FREITAS, M.; MACHADO, B. J.; TRISTÃO, L.; RUGGERI, J. Tendências observadas em indicadores de extremos climáticos de temperatura e precipitação no estado do Paraná. Revista Brasileira de Meteorologia, v. 30, n. 2, p. 181-194, 2015. https://doi.org/10.1590/0102-778620130622.

SOBRINO, J. A.; GOMEZ, M.; JIMENEZMUNOZ, J. C.; OLIOSO, A. Application of a simple algorithm to estimate daily 
evapotranspiration from NOAA-AVHRR images for the Iberian Peninsula. Remote Sensing of Environment, v. 110, p. 139148 , 2007. https://doi.org/10.1016/j.rse.2007.02.017

SOUZA, A. P.; MOTA, L. L.; ZAMADEI, T.; MARTIN, C. C.; ALMEIDA, F. T.; PAULINO, J. Classificação climática e balanço hídrico climatológico no estado de Mato Grosso. Nativa, v. 1, n. 1, p. 34-43, 2013. http://dx.doi.org/10.14583/23187670.v01n01a07

TANK, A. M. G. K.; ZWIERS, F. W.; ZHANG, X. Guidelines on Analysis of extremes in a changing climate in support of informed decisions for adaptation. World Climate Data and Monitoring Programme (WCDMP): 2009. $72 \mathrm{p}$.

TASUMI, M.; ALLEN, R. G.; TREZZA, R. Atsurface reflectance and albedo from satellite for operational calculation of land surface energy balance. Journal of Hydrologic Engineering, v. 13, p. 51-63, 2008. https://doi.org/10.1061/(ASCE)10840699(2008)13:2(51)

USGS - United States Geological Survey. EROS Science Processing Architecture (ESPA). $2018 . \quad$ Disponível em: $<$ https://espa.cr.usgs.gov>. Acesso em: $06 \mathrm{de}$ julho de 2018.

WANG, X. L.; WEN, Q. H.; WU, Y. Penalized maximal $t$ test for detecting undocumented mean change in climate data series. Journal of Applied Meteorology and Climatology, v. $\quad 46, \quad$ n. $\quad 6, \quad 2007$. https://doi.org/10.1175/JAM2504.1

WANG, X. L. Accounting for Autocorrelation in Detecting Mean Shifts in Climate Data Series Using the Penalized Maximal $t$ or $F$ test. Journal of Applied Meteorology and Climatology, v. 47, p. 2423-2444, 2008. https://doi.org/10.1175/2008JAMC1741.1

ZHANG, X.; YANG, F. RClimDex (1.0) User Guide. Climate Research Branch Environment Canada. Ontario: Downsview; 2004. $22 \mathrm{p}$. 